\title{
The controlled clinical trial and its application to neglected healthcare problems in Sri Lanka
}

\author{
Asita de Silva ${ }^{1}$ \\ Journal of the Ceylon College of Physicians, 2012, 43, 15-19
}

\section{General Introduction}

Deliberate experiments designed to assess upon patients the value of therapeutic procedures are an essential feature of modern medicine. These clinical trials link theoretical considerations and laboratory investigation with clinical practice and so provide a reliable basis for advances in treatment. They are seldom easy, usually costly of time and effort and sometimes dangerous to patients. The danger is twofold: it may be harmful to patients treated in the trial, or to patients badly treated as a result of reliance upon an inadequate trial. The efficiency of these experiments is thus a matter of great importance.

In the era of evidence-based medicine there are an ever increasing number of treatment innovations which require rigorous investigation to see if they are of genuine benefit. The evaluation of possible improvements in the treatment of disease has, historically, been an inefficient and haphazard process. Only in recent years has it become widely recognized that properly conducted clinical trials, which follow the principles of scientific experimentation, provide the only reliable basis for evaluating the efficacy and safety of new treatments and evaluation of treatment effect on patients.

As historians review the advances of therapeutic science in the second half of the 20th century, one of the outstanding methodologic achievements will surely be the use of evidence from randomised trials to show the efficacy of new therapeutic agents. With this type of evidence, clinicians and patients can now be reasonably reassured that, for the first time in medical history, a new treatment actually does what is claimed for it.

A clinical trial can be simply defined as a planned experiment on patients with a given medical condition, designed to elucidate their most appropriate treatment. The essential characteristic of a clinical trial is that

\footnotetext{
${ }^{1}$ Director, Clinical Trials Unit, and Professor, Department of Pharmacology, Faculty of Medicine, University of Kelaniya, Sri Lanka.
}

one uses results based on a limited sample of patients to make inferences about how treatment should be conducted in a more generalised population of patients who will require treatment in the future. The great majority of clinical trials are concerned with the evaluation of drug therapy. However, clinical trials may also be concerned with other forms of treatment. For example, surgical procedures, different forms of medical advice (e.g. diet and exercise policy after myocardial infarction), and even alternative approaches to patient management (e.g. general ward or stroke unit care after cerebrovascular accident).

\section{Evidence in toxicology and toxinology}

In 1996 an editorial in the journal, Clinical Toxicology, stated explicitly what most clinicians knew, that it was not possible to practice 'evidence based medicine' in clinical toxicology ${ }^{1}$. This is because there was little evidence: the published data on treatment included very few randomised clinical trials addressing clinically important end-points. Observational studies of clinical features were lacking in both quantity and quality, and diagnostic and prognostic criteria were not prospectively validated. As a result, knowledge and management of many forms of poisoning was based largely on case reports, and clinicians relied on textbooks and expert opinion for guidance ${ }^{1}$.

Ironically, the World Health Organization (WHO) estimates that more than $600-700,000$ people die of poisoning and envenoming each year in Asia and the Western Pacific ${ }^{2}$. The in-hospital mortality rate following poisoning and envenoming is high in developing countries, approaching $15 \%$ in Sri Lanka, compared with less than $1 \%$ in the United Kingdom ${ }^{3,4}$. While primary prevention has great potential in the long term to reduce the number of deaths, improving medical management based on sound evidence will also reduce the death rate and probably do it much faster.

Current protocols for the management of poisoning are based on little evidence and are difficult to deliver in the resource-poor settings in which most poisonings occur. The in-hospital case fatality rate for poisoning in the developing world is $10-20 \%$ or more, 
while in the developed world it is well under $0.5 \%$. This glaring disparity by itself underlines the importance of developing the most cost-effective therapeutic interventions to our healthcare problems. Thus, the need and potential for high quality clinical research in poisoning and envenoming is enormous. Management dilemmas associated with yellow oleander poisoning and safety of antivenom after snakebite envenomation, which cause significant morbidity and mortality particularly among the farming communities of this country are two such areas that needed urgent attention.

Between 1986 and 2000, the total number of hospital admissions due to poisonings nationally, doubled $^{5}$. Although the majority of these poisonings is due to pesticide poisoning, there are now tens of thousands of yellow oleander poisoning cases in Sri Lanka and South Asia contributing to these morbidity figures each year, and probably thousands of deaths. Management of patients with serious oleander poisoning is difficult and costly, placing great stress on the healthcare system due to requirements for expensive treatments and transfers to secondary or tertiary hospitals for temporary cardiac pacing and intensive care.

In addition, snakebite is a major cause of mortality and morbidity, and it has a significant impact on human health and the economy through treatment related expenditure and loss of productivity ${ }^{6,7}$. As a neglected public health problem, estimates of the risk is largely unknown. However, the associated personal and economic impact of snakebite is substantial across developing countries including Sri Lanka. Antivenoms, the only specific treatment for snakebite envenoming, have been available in South Asia for the past 60 years, with all existing products manufactured in India. While their efficacy in restoring haemostasis and cardiovascular functions after envenomation is well established, up to $80 \%$ of patients treated with these Indian antivenoms develop one or more adverse effect such as anaphylactoid or pyrogenic reactions, or late serum sickness ${ }^{8,9}$. Sadly, most of the antivenoms that are routinely used in South Asia have never been subjected to independent preclinical testing and formal evaluation in clinical trials. Their safety profiles have not been properly established, and there is currently no evidence-based protocol for interventions aimed at improving safety?

There are many potential interventions with different mechanisms that could prove to be useful for these clinical problems. Although large amounts of money are spent on antidote drug development in Western laboratories, no funding is extended to conduct clinical trials to address these issues. Clearly, these unique healthcare challenges faced by our clinicians in their day-to-day practice of medicine require home-grown answers.

Most clinical research in Sri Lanka before the mid 1990s centered around observational studies and retrospective analyses. These, obviously, could not contribute to an evidence base that would change clinical practice. As a result, too little evidence was being generated where it was required most. However, there has subsequently been a shift in the methodology used for generating evidence in poisoning and envenoming with many groups starting to conduct interventional clinical research. Most of these clinical trials have been on organophosphate and other pesticide poisoning, but some have attempted to address problems related to yellow oleander poisoning and antivenom safety.

Several small clinical trials using different methods have been conducted to reduce acute adverse reactions to antivenom. In one study of 105 patients, low-dose adrenaline given subcutaneously immediately before administration of antivenom to snakebite victims significantly reduced the incidence of acute adverse reactions to the serum ${ }^{9}$. However, the investigators stopped recruitment halfway through the study when an ad-hoc interim analysis showed a significant reduction in all acute adverse reactions when adrenaline was used as pretreatment rather than placebo. Unfortunately, in a subanalysis of the severity of adverse reactions in the two groups the differences in mild, moderate, and severe reactions barely reached significance due to the low power of the analysis (about $50 \%$ ) and the small numbers randomised. This is important since it is particularly the moderate and severe reactions that we wish to prevent. As it was, the trial was stopped because of a supposed benefit, but the trial could not provide convincing evidence on the value of subcutaneous adrenaline in the prevention of reactions to antivenom. Furthermore, the study was unable to enroll sufficient participants to adequately establish safety, a major concern in the use of adrenaline in a prophylactic role $^{10}$, particularly the risk of intra-cerebral haemorrhage ${ }^{11,12}$. Thus, the lack of preplanned interim analyses built into the trial methodology at the outset precluded the results of this otherwise well conducted clinical trial from producing conclusive evidence for policy recommendations.

A more recent study suggested that intravenous hydrocortisone is ineffective in preventing acute adverse reactions to antivenom, but if given together with intravenous chlorpheniramine it may reduce these reactions ${ }^{13}$. However, this trial recruited only 52 patients and was not designed to study the efficacy of 
chlorpheniramine alone, making decisive interpretation of the results and recommendations on pretreatment with steroids and antihistamines to prevent acute reactions to antivenom difficult.

The clinical features of yellow oleander poisoning are similar to those of poisoning with digitalis and other cardiac glycosides. Therefore, despite its very high cost Anti-digoxin Fab antitoxin is considered by some to be the gold standard of therapy for yellow oleander poisoning. However, the only placebo-controlled clinical trial of Anti-digoxin Fab antitoxin did not evaluate improvements in mortality, and was not powered to assess this most important endpoint ${ }^{14}$. Although the antitoxin showed benefit in improving cardiac rhythm, there were no deaths in both treatment and placebo arms of this study ${ }^{14}$. The same group subsequently conducted an open label study of multiple-dose activated charcoal in three secondary referral hospitals and reported that multiple-dose activated charcoal did not reduce mortality in patients with yellow oleander poisoning ${ }^{15}$ compared to a single dose of activated charcoal. However, a closer analysis of results showed that these investigators had not randomised sufficient numbers to arrive at this conclusion. The sample size for this trial was calculated to detect whether multipledose activated charcoal can reduce case fatality from $10 \%$ to $7 \%$ with a significance of $5 \%(p=0.05)$ and a power of $80 \%{ }^{15}$. However, the mortality in the control group in this trial was only $4.7 \%$, and not $10 \%$ as expected. Therefore, to detect a comparable 30\% reduction in the actual event rate from $4.7 \%$ to $3.3 \%$ they would have required more than 3000 patients in each group in the study. With the numbers they reported for the multiple-dose and single-dose groups of 541 and 549, respectively, their study would have had a power of only $18 \%$ to detect such a difference; a clear example of a clinical trial losing its way and not producing the anticipated results due to lack of preplanned interim analyses to guide the study investigators.

As illustrated with some examples of local clinical trials (above) most of these studies were hampered by lack of clear apriori hypotheses, multi-disciplinary investigator teams, data safety monitoring and preplanned interim analyses, small sample sizes resulting in inadequate power, unclear endpoints, and other methodological hallmarks of poor trial design. As a result, the findings of these trials were largely inconclusive making clear recommendations for management protocols difficult.

Furthermore, because of the nature of the clinical questions addressed by clinical trials in these areas of tropical medicine, it is important to have a robust pragmatic approach to the design of studies. In a pragmatic approach, a therapeutic trial is intended to ask and answer questions that are directly cogent for the "messy" realities of clinical practice. The groups under study represent the heterogeneous patients seen in practice; the treatments outside the trial protocol are given in the manner employed by practicing physicians; and the results are analyzed according to what actually happened. Most early clinical trials in Sri Lanka lacked this approach and took a more "fastidious" approach that may have resulted in patients in the study groups being relatively homogeneous. That makes the generalisability of results of such clinical trials to patient populations in "real life" clinical practice more difficult.

The modern clinical trial is sophisticated and is expected to address these design and methodological issues at the outset. This necessitates a structured approach by the investigators. Conduct of such clinical trials require a multidisciplinary team of investigators, wide collaborations, clear definition of trial endpoints, large sample sizes for adequate powering, guidance from data safety monitoring and steering committees, pre-planned interim analyses and termination rules, a pragmatic approach to design and execution, and if possible disease markers to support clinical findings.

The clinical trials on yellow oleander poisoning and improving antivenom safety described in this oration were designed and conducted taking into account methodological issues of modern clinical research. These large clinical trials were done through a dedicated academic clinical trials centre at the Faculty of Medicine, University of Kelaniya, in collaboration with many State hospitals and international centres of excellence in the Universities of Oxford and Liverpool. They highlight the evolution of clinical trials in tropical medicine in this country.

\section{General Conclusions}

The practicality, need for, and essential characteristics of the modern controlled trial has been debated and even tentatively tested progressively over at least three centuries and several major changes have been made in the last 50 years to the 1948 model. The evolution of the clinical trial has been a slow and spluttering process that was speeded up in 1948, just as the evolution of homo sapiens that occurring over several millions of years may have been qualitatively changed by a mutation in a single gene that facilitated the development of language about $100-150,000$ years ago $^{16}$.

Over the past half century there has been a vast proliferation, first of randomized controlled trials and now of meta-analyses, both of which reduce bias. 
These have helped to bring about a situation in which advances in healthcare have been credited with six of the ten years of increased life expectancy over that time period and an average of seven additional years of partial or complete relief from the poor quality of life associated with chronic disease ${ }^{17}$. However, to get medically reliable answers to previously unanswered questions about life or death treatment decisions, it is not just enough to avoid bias. We must ensure that we are not misled by the play of chance, and often the only way to do this reliably is to get appropriate analyses of really large scale randomised evidence.

The consequences of not conducting appropriate clinical trials at the proper time can be serious or costly. An example was the uncertainty as to the efficacy and safety of digitalis in congestive heart failure. Only in the 1990's, after the drug had been used for over 200 years, was a large clinical trial evaluating the effect of digitalis on mortality mounted ${ }^{18}$. High concentration oxygen was administered to premature infants until clinical trial evidence demonstrated it caused blindness ${ }^{19}$. A clinical trial can also determine the incidence of adverse effects or complications of the intervention. Few interventions, if any, are entirely free of undesirable effects. However, drug toxicity might go unnoticed without the systematic follow-up measurements obtained in a clinical trial of sufficient size. The Cardiac Arrhythmia Suppression Trial documented that commonly used antiarrhythmic drugs were harmful in patients who had a history of myocardial infarction, and raised questions about routine use of an entire class of antiarrhythmic agents ${ }^{20}$. Corticosteroids had been commonly used to treat people with traumatic brain injury. Small clinical trials were inconclusive, and a meta-analysis of 16 trials showed no difference in mortality between corticosteroids and control ${ }^{21}$. Because of this uncertainty a large clinical trial was conducted. This trial, with far more participants than the others combined, demonstrated a significant 18\% relative increase in mortality at 14 days $^{22}$ and a $15 \%$ increase at 6 months $^{21}$. As a result, a plotting of the meta-analysis recommended against the routine use of corticosteroids in people with head injury ${ }^{23}$.

At present many at best unreliable and at worst wrong answers to important healthcare problems in Sri Lanka are being generated by non-randomised outcome research, by underpowered poorly designed trials, by audits and retrospective studies, and by statistically inappropriate analyses. The use of antivenom after snakebite envenomation is a case in point. While their efficacy in restoring haemostasis and cardiovascular functions is well established, the ability of antivenoms to prevent tissue damage and to reverse neurotoxicity is more controversial24,25. For instance, administration of antivenom to krait bite victims with established respiratory paralysis does not reverse paralysis ${ }^{25,26}$. This lack of clinical effectiveness often contributes to the administration of excessive amounts of antivenom and the potential to cause harm $^{27,28}$. In fact, there is currently no evidence-based protocol for their administration and dosage ${ }^{29}$. As such, there is an urgent need to conduct well designed clinical trials to address these fundamental issues, particularly dose finding studies to delineate the appropriate dosage and administration regimen for the locally available polyvalent antivenom, in snakebite management.

The requirement in pesticide and other poisonings is similar. There are many potential antidotes and therapies with many different mechanisms that have shown synergistic benefit in animal models. Unfortunately, all have failed to progress to clinical trials. It is not surprising, therefore, that systematic reviews suggest there is no good quality human evidence that any currently used antidote, other then atropine, is of benefit ${ }^{30}$.

Over the past six to eight years, our group has sought to address some important healthcare problems in this country, particularly in snakebite and poisoning, by conducting large well-designed clinical trials. Our results have shown that pragmatic clinical trials that are well designed and adequately powered can find cost effective solutions to at least some of the major healthcare problems that are unique to us. These findings have changed clinical practice, which is what clinical trials should do. Furthermore, the work described here unequivocally demonstrates the value of addressing clinical questions through large randomized clinical trials and the value of collaborating with clinicians and other centres of excellence.

Several developments could help to ensure that efforts over the next 50 years will be more effective in yielding unbiased, relevant, and reliable assessments of the effects of healthcare. Information derived from systematic reviews of past research and from registers of continuing clinical trials will help to highlight where new trials are ongoing and where they are still needed and how best to maximise the quality and relevance of the new information sought. The millions of poisoning and envenomings occurring in the developing world each year offer immense opportunities for clinical research. We need to make use of these opportunities to design and conduct much simpler and much larger clinical trials. Otherwise the next 50 years of randomised evidence will not fulfill the promise of 50 years ago, when the properly randomised clinical trial was first published, transforming medical research by its method of generating unbiased answers to many pivotal therapeutic questions. 


\section{References}

1. Buckley NA, Smith AJ. Evidence-based medicine in toxicology: where is the evidence? Lancet 1996; 347(9009): 1167-9.

2. Annex Table 2 Deaths by Cause, Sex and Mortality Stratum in WHO Regions, Estimates for 2001. World Health Report 2002. Geneva: World Health Organization, 2002.

3. Eddleston M, Sheriff MHR, Hawton K. Deliberate self-harm in Sri Lanka: an overlooked tragedy in the developing world. BMJ 1998; 317: 133-5.

4. Hettiarachchi J, Kodithuwakku GCS. Self-poisoning in Sri Lanka: factors determining the choice of the poisoning agent. Human Toxicology 1989; 8: 507-10.

5. Buckley NA, Karaliedde L, Dawson A, Senanayake N, Eddleston $\mathrm{M}$. Where is the evidence for treatment used in pesticide poisoning? Is clinical toxicology fiddling while the developing world burns? Clinical Toxicology 2004; 42 : 113-16.

6. Rahman R, Faiz MA, Selim S, Rahman B, Basher A, et al. (2010) Annual Incidence of Snake Bite in Rural Bangladesh. PLoS Negl Trop Dis 4(10): e860. doi:10.1371/journal. pntd. 0000860.

7. Alirol E, Sharma SK, Bawaskar HS, Kuch U, Chappuis F (2010) Snake Bite in South Asia: A Review. PLoS Negl Trop Dis 4(1): e603. doi:10.1371/journal.pntd.0000603.

8. Ariaratnam CA, Sjostrom L, Raziek Z, Kularatne SA, Arachchi $\mathrm{RW}$, et al. An open, randomised comparative trial of two antivenoms for the treatment of envenoming by Sri Lankan Russell's viper (Daboia russelii russelii). Trans R Soc Trop Med Hyg 2001; 95: 74-80.

9. Premawardhena AP, de Silva CE, Fonseka MM, Gunatilake $\mathrm{SB}$, de Silva HJ. Low dose subcutaneous adrenaline to prevent acute adverse reactions to antivenom serum in people bitten by snakes: randomised, placebo controlled trial. BMJ 1999; 318: 1041-3.

10. Khanna R, Hawkins WJ (1999). A plea for caution in the use of adrenaline (1999) BMJ (electronic version) bmj.com: 19 May.

11. DassanayakeAS, Karunanayake P, Kasturiratne KT, Fonseka MMD, Wijesiriwardena B, et al. Safety of subcutaneous adrenaline as prophylaxis against acute adverse reactions to anti-venom serum in snakebite. CMJ 2002; 47: 48-9.

12. Horowitz BZ, Jadallah S, Derlet RW. Intracranial bleeding associated with prehospital use of epinephrine. Ann Emerg Med 1996; 28: 725-7.

13. Gawarammana IB, Abeysinghe S, Kularatne M, Dissanayake WP, Kumarasiri RPV, et al . Parallel infusion of hydrocortisone chlorpheniramine bolus injection to prevent acute adverse reactions to antivenom for snakebites: a randomised, double-blind, placebo-controlled study. Med J Aust 2004; 180: 20-3.

14. Eddleston M, Rajapakse S, Rajakanthan, Jayalath S, Sjöström L, Santharaj W, et al. Anti-digoxin Fab fragments in cardiotoxicity induced by ingestion of yellow oleander: a randomised controlled trial. Lancet 2000; 355: 967-72.

15. Eddleston M, Juszczak E, Buckley NA, Senarathna L,
Mohamed F, Dissanayake W, et al. Multiple-dose activated charcoal in acute self-poisoning: a randomised controlled trial. Lancet 2008; 371: 579-87.

16. Sargent CA, Blanco $P, A f f a r a ~ N A$. Did the hominid specific regions of $X-Y$ homology contain candidate genes potentially involved in a critical event linked to specification? In: The Speciation of modern Homo Sapiens. 2002, Ed. Crow TJ, Oxford University Press, Oxford.

17. Royal College of Physicians of Edinburgh/UK Cochrane Centre. Controlled trials from history. www.rcpe.ac.uk/ cochrane.

18. Smith TW. Digoxin in heart failure. N Engl J Med 1993; 329: 51-3.

19. Enhorning G, Shennan A, Possmayer F, Dunn M, Chen CP, Milligan $\mathrm{J}$. Prevention of neonatal respiratory distress syndrome by tracheal instillation of surfactant: a randomised clinical trial. Paediatrics 1985; 76: 145-53.

20. RJ Myerburg, KM Kessler, RJ Prineas, et al. The Cardiac Arrhythmia Suppression Trial. N Engl J Med 1989; 321: 1754-6.

21. Giannotta SL, Weiss MH, Apuzzo ML, Martin E. High dose glucocorticoids in the management of severe head injury. Neurosurgery 1984; 15: 497-501.

22. Edwards P, Arango M, Balica L, Cottingham R, El-Sayed H, Farrell $\mathrm{B}$, et al. Final results of MRC CRASH, a randomised placebo-controlled trial of intravenous corticosteroid in adults with head injury-outcomes at 6 months. Lancet 2005; 365: 1957-9.

23. Alderson P, Roberts I. Corticosteroids for acute brain injury. Cochrane Database of Systematic Reviews 2005, Issue 1. Art. No.: CD000196. DOI: 10.1002/14651858.CD000196.pub2

24. Kularatne SA. Common krait (Bungarus caeruleus) bite in Anuradhapura, Sri Lanka: a prospective clinical study, 199698. Postgrad Med J 2002; 78: 276-80.

25. Theakston RD, Phillips RE, Warrell DA, Galagedera Y, Abeysekera DT, et al. Envenoming by the common krait (Bungarus caeruleus) and Sri Lankan cobra (Naja naja naja): efficacy and complications of therapy with Haffkine antivenom. Trans R Soc Trop Med Hyg 1990; 84: 301-8.

26. Bawaskar HS, Bawaskar PH. Profile of snakebite envenoming in western Maharashtra, India. Trans $R$ Soc Trop Med Hyg 2002; 96: 79-84.

27. Sharma N, Chauhan S, Faruqi S, Bhat P, Varma S. Snake envenomation in a north Indian hospital. Emerg Med J 2005; 22: 118-20.

28. Sharma SK, Koirala S, Dahal G. Krait bite requiring high dose antivenom: a case report. Southeast Asian J Trop Med Public Health 2002; 33: 170-1.

29. Alirol E, Sharma SK, Bawaskar HS, Kuch U, Chappuis F (2010) Snake Bite in South Asia: A Review. PLoS Negl Trop Dis 4(1): e603. doi:10.1371/journal.pntd.0000603

30. Buckley NA, Karaliedde L, Dawson A, Senanayake N, Eddleston $\mathrm{M}$. Where is the evidence for treatment used in pesticide poisoning? Is clinical toxicology fiddling while the developing world burns? Clinical Toxicology 2004; 42: 113-16. 